\title{
Impact of MRCP findings on the management of biliary strictures in post- living donor liver transplant
}

\author{
Mohamed Saied Abdelgawad ${ }^{1 *}$, Rasha Abdelhafiz Aly ${ }^{1}$ and Ahmed Elshawadfy Sherif ${ }^{2}$
}

\begin{abstract}
Background: Biliary strictures are a well-recognized complication of liver transplant. Magnetic resonance cholangiopancreatography (MRCP) is the imaging modality of choice for the diagnosis of post-liver transplant biliary strictures because of its high rate of diagnostic accuracy and limited risk of complications. The purpose of this study was to assess the potential role of MRCP in the diagnosis and management of biliary strictures following living donor liver transplantation (LDLT). Data were retrospectively collected from recipients who had developed biliary stricture following living donor liver transplantation. Informed consent was signed by the patients. All patients were subjected to a proper assessment of history and clinical examination as well as routine laboratory investigations. MRCP was performed to diagnose biliary strictures and to follow cases after management.

Results: Analysis involved 32 liver transplant recipients as 28 had anastomotic biliary stricture, 2 had intrahepatic biliary stricture, and 2 had both types (mixed biliary stricture). Management was done in 20 patients by endoscopic therapy and 4 patients by surgical therapy, while 8 patients were exposed only for long-term follow-up. Satisfactory improvement of the biliary stricture was noted in about 16 with endoscopic therapy and 2 with surgical therapy.
\end{abstract}

Conclusion: MRCP is a reliable non-invasive imaging modality for evaluating the biliary stricture following LDLT providing important information on its management.

Keywords: Liver transplantation, Biliary stricture, Magnetic resonance cholangiopancreatography

\section{Background}

Biliary complications following liver transplantation represent a common source of morbidity despite advances in surgical techniques, occurring early (within 30 days of transplant) or late [1]. Biliary strictures are a common complication and account for around 35\% of all biliary complications of liver transplant and these result in decreased patient survival [2-4]. The factors that most commonly contribute to stricture formation include the surgical reconstruction technique (e.g., duct-to-duct anastomosis vs choledochojejunostomy); The use of a Ttube and the type of liver transplant procedure (LDLT) are more prone to strictures than deceased donor liver

\footnotetext{
* Correspondence: mselgawad@yahoo.com

${ }^{1}$ Radiology Department - National Liver Institute, Menoufia University,

Menuofia, Egypt

Full list of author information is available at the end of the article
}

transplantation (DDLT) and to the development of hepatic arterial thrombosis [5].

Biliary strictures are classified as anastomotic or nonanastomotic strictures, depending on their location [5]. Biliary strictures can occur months to years after liver transplantation, but they most commonly present within the first year, with a mean interval from transplant to time of presentation of 5 to 8 months [6,7]. Strictures that occur early after liver transplant usually result from technical problems in the surgery itself, whereas strictures that develop later arise mainly from vascular insufficiency, immunologic causes, or problems with healing and fibrosis $[8,9]$.

The anastomotic biliary strictures (ASs) or non-anastomotic biliary strictures (NASs) differ in terms of pathophysiology, clinical course, and response to therapeutic interventions. ASs tend to be single, short in length, and localized to the site of biliary anastomosis, 
Table 1 Summary of magnetic resonance cholangiopancreatography imaging parameters

\begin{tabular}{lllllll}
\hline Sequence & TR $(\mathrm{ms})$ & $\begin{array}{l}\text { TE } \\
(\mathrm{ms})\end{array}$ & $\begin{array}{l}\text { Thickness } \\
(\mathrm{mm})\end{array}$ & $\begin{array}{l}\text { Gap } \\
(\mathrm{mm})\end{array}$ & $\begin{array}{l}\text { FOV } \\
(\mathrm{cm})\end{array}$ & Matrix \\
\hline $\begin{array}{l}{ }^{*} \text { Cor T2 RTr } \\
\text { PROP }\end{array}$ & 2727 & 98 & 5 & 1 & 38 & $320 \times$ \\
*Axial T2 FSE & 2000 & 95 & 5 & 1.5 & 38 & $320 \times$ \\
$\begin{array}{l}\text { RTr PROP } \\
\text { *Cor 3D MRCP }\end{array}$ & $3000-$ & $500-$ & 1 & 0 & 36 & $288 \times$ \\
RTr & 5000 & 1000 & & & & 288 \\
*Axial 3D thin & $2000-$ & $400-$ & 2 & 0 & 36 & $224 \times$ \\
Cut RTr & 5000 & 1000 & & & & 224 \\
*DWl & 8700 & $50-$ & 5 & 1.5 & 38 & $96 \times$ \\
& & 200 & & & & 128 \\
\hline
\end{tabular}

$T R$, repetition time; $T E$, echo time; FOV, field of view; $R T$, respiratory trigger; $P R O P$, propeller family introduction; $F S E$, fast spin echo; $3 D$, three-dimensional; $D W I$, diffusion weighted image

whereas NAS are more likely to be multiple and longer and are often intrahepatic or in the donor duct proximal to the anastomotic site $[1,10]$. NASs occur less frequently and earlier than ASs with a mean time to stricture formation of 3 to 6 months and are more likely to result in graft loss [5].

Ultrasound is considered the first imaging modality for LT recipients with suspected biliary complications. LT recipients with biliary strictures develop varying degrees of biliary duct dilatation due to denervation of the sphincter of Oddi and the biliary tract leading to increased intra-choledochal pressures. A limitation of ultrasound (US) is that it lacks dynamic panoramic assessment of the biliary tree, thereby contributing to its poor sensitivity [11].

According to several studies, magnetic resonance cholangiopancreatography (MRCP), a technically advanced non-invasive imaging modality, provides a panoramic detailed presentation of the biliary tree that enables the

\section{MRCP grading of biliary strictures}

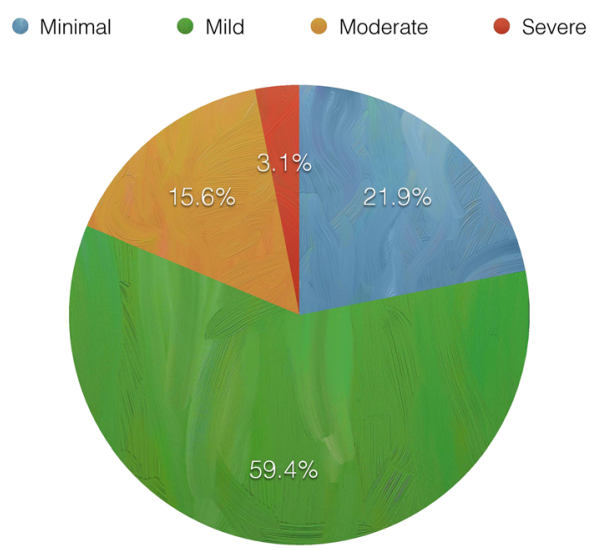

Fig. 1 MRCP grading of biliary strictures clinicians to identify the type, site, and degree of the biliary problem [12]. MRCP has a sensitivity and specificity of $85-90 \%$ in diagnosing post-liver transplant biliary strictures [13]. MRCP can also offer a road map for the endoscopist in planning the necessary intervention [14]. Another advantage of MRCP is that it does not carry the invasive risk that occur with endoscopic retrograde cholangiopancreatography (ERCP) or other interventions such as percutaneous transhepatic cholangiography (PTC) [15].

Advances in endoscopic therapy and interventional radiology have a potential role in decreasing the need for surgical repair or retransplant, both of which carry much higher morbidity and mortality rates. Currently, the first line of treatment for biliary strictures is endoscopic therapy [5]. In this article, we discuss the classification of post-liver transplant biliary strictures and their diagnosis by MRCP and its impact on the management strategy.

\section{Methods \\ Patient characteristics}

Review and retrospective analysis of the health records of adult living donor liver transplantation recipients referred to the MRI unit in the National Liver Institute, Menoufia University between January 2016 and December 2018.

Review of their medical charts revealed that the indication for transplantation was hepatitis $\mathrm{C}$ virus $(\mathrm{HCV})$ cirrhosis in 17 patients, hepatitis B virus (HBV) cirrhosis in two, hepatocellular carcinoma (HCC) in ten, primary sclerosing cholangitis (PSC), secondary biliary cirrhosis, and Wilson's disease in one each. Of our 32 cases, 30 underwent biliary reconstruction by choledochocholedochostomy with only one case revealing difficulty in intraoperative biliary anastomosis followed by successful duct reanastomosis, while the other two underwent biliaryenteric reconstruction (choledochojejunostomy).

Only recipients who developed post-transplant biliary strictures were selected. A written consent was signed by all patients or their relatives before the procedure. The study was approved by the Research Ethics Committee of the National Liver Institute.

Biliary stricture was diagnosed based on clinical, laboratory, and imaging studies (including ultrasound and MRCP). They were referred for MRCP study of the biliary system to confirm the presence of stricture, its level, and grades.

All patients were subjected to the following: clinical evaluation; laboratory examinations including serum bilirubin, liver enzymes (aspartate transaminase and alanine transaminase), alkaline phosphatase, and $\gamma$-glutamyl transferase; and imaging investigations including US with colored Doppler and MRCP. 


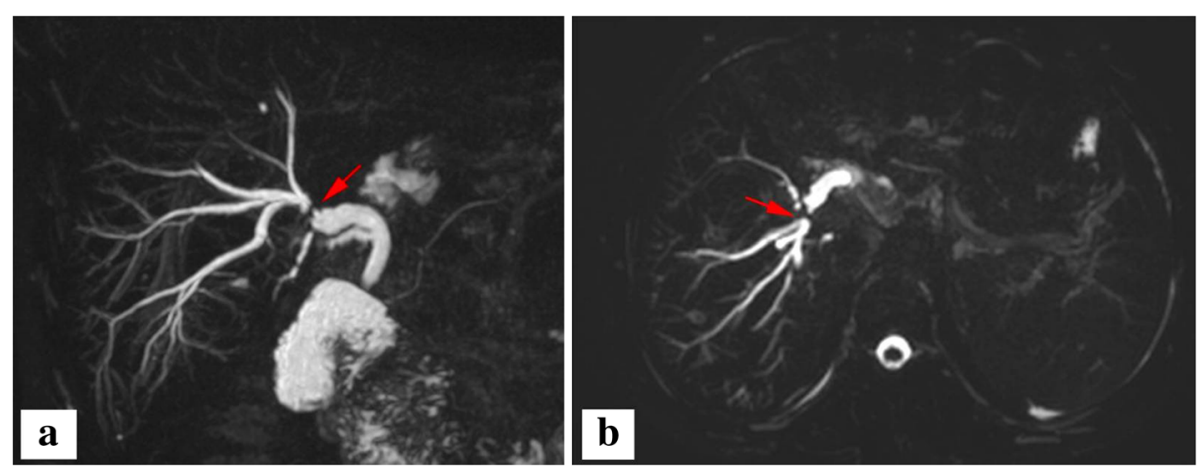

Fig. 2 A 34-year-old female 5 months after liver transplantation with duct-to-duct anastomosis. MRCP 3D MIP reconstructed coronal (a) and axial (b) images showed the presence of anastomotic biliary stricture (red arrow) with mild intrahepatic biliary dilatation.

\section{MRCP technique}

Patients were asked to fast for $8 \mathrm{~h}$ prior to the study in order to reduce fluid secretions within the stomach and duodenum and reduce small bowel peristalsis. MRCP was performed using a 1.5-T MRI system (GE, Optima $450 \mathrm{~W}, 32$ channels), using a phased-array body coil. The protocol imaging parameters are shown in Table 1.

\section{MRCP diagnosis and grading of biliary stricture}

Specific criteria have been proposed for stricture diagnosis on MRCP imaging (Fig. 1). The type of biliary anastomosis, length of stricture, and differential diameters of recipient and donor ducts were needed to be studied in MRCP. Anastomotic biliary stricture (ABS) is diagnosed on MRCP by the reduction in the anastomotic diameter with upstream dilatation of the donor liver allograft biliary radicles. The presence of stricture intrahepatically is to be considered alone as narrowing proximal to the anastomotic site within the intrahepatic biliary branches with consequent localized distal biliary dilatation (intrahepatic/non-anastomotic stricture) or in combination with the anastomotic stricture (mixed type).
If biliary stricture is diagnosed, stricture grading was done as minimal, mild, moderate, and severe strictures according to the degree of intrahepatic (donor) bile duct dilatation. The determination of site and grading of post-transplant biliary stricture has implications on the plane of therapy as well as the patient's outcome. After their initial MRCP examination, our patients were followed up for a long term over a period of 36 months until December 2018 or until death occurred.

\section{Management}

When a clinically significant stricture is found, treatment is necessary. Recipients with post-transplant biliary strictures were managed with endoscopic retrograde cholangiopancreatography (ERCP) as b dilatation was done with or without plastic stent insertion in 20 patients and surgical intervention in 4 patients by the mean of Rouxen-Y biliary-enteric reconstruction. Improvement of symptoms of biliary stricture was proven clinically and with follow-up MRCP.

\section{Statistical analysis}

The data collected were tabulated and analyzed by the Statistical Package for Social Science (SPSS) version

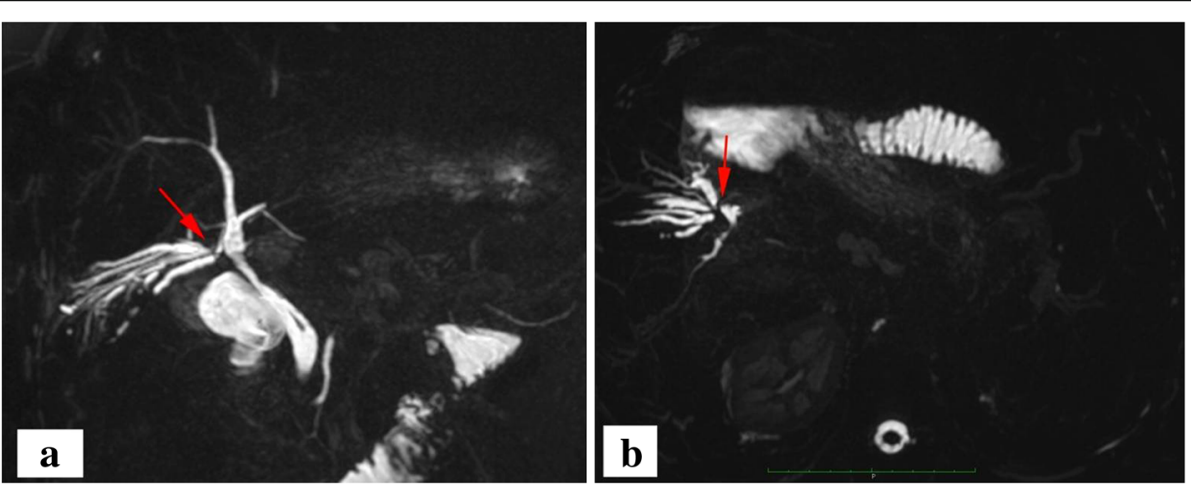

Fig. 3 A 50-year-old male 15 months after liver transplantation with duct-to-duct anastomosis. MRCP 3D MIP reconstructed coronal (a) and axial (b) images showed the presence of intrahepatic biliary stricture (red arrow) 


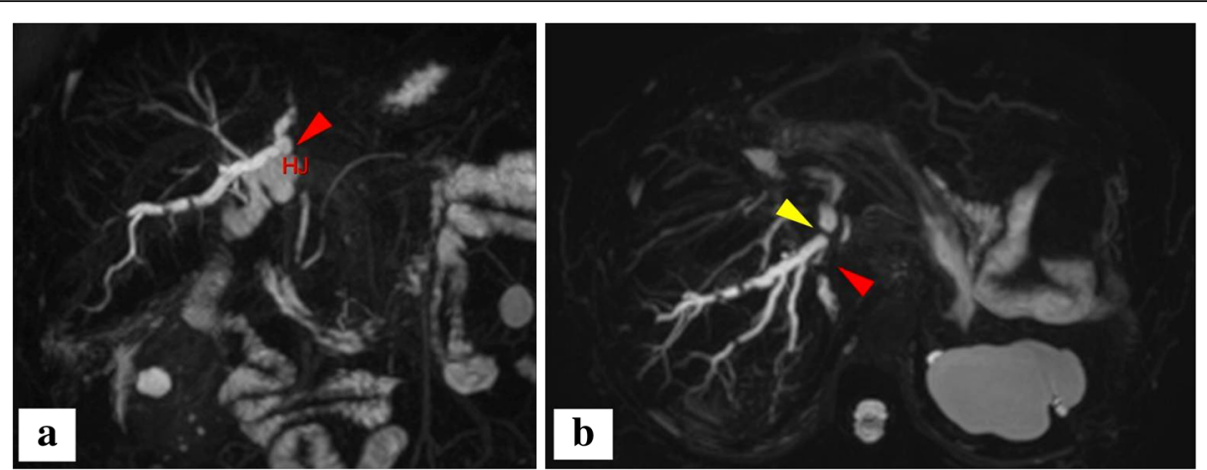

Fig. 4 A 60-year-old male 20 months after liver transplantation with hepaticojejunostomy (biliary-enteric anastomosis). MRCP 3D MIP reconstructed coronal (a) image showed the presence of anastomotic stricture (red arrowhead) and axial (b) image showed both anastomotic (yellow arrowhead) and intrahepatic stricture involving donor posterior bile duct (red arrowhead)

25.0. Parametric data were presented with mean \pm standard deviation and non-parametric data with median and range. Categorical data were presented with frequency and percentage. Correlation between the degree of biliary stricture and the clinical outcomes of the recipients after treatment will be done with chi-square or Fisher's exact test as appropriate.

\section{Results}

This study was performed on 32 (26 males and 6 females) adult LDLT recipients with mean age 50.53 years ranging between 27-65 years old.

Biliary stricture was found early within 3 months postoperative in 10 patients $(31.2 \%)$, while the other 22 patients $(68.8 \%)$ revealed biliary stricture later than 3 months.

Of total cases, anastomotic strictures (Fig. 2) were seen on MRCP in 28 recipients, while two were included in the intrahepatic stricture type (Fig. 3) and two in the mixed type (both biliary strictures) (Fig. 4) (Table 2).

The degree of biliary stricture of the studied cases were classified by MRCP into the following: minimal stricture in 7 out of 32 patients (21.9\%), mild stricture in 19 out of 32 patients (59.4\%), moderate stricture in 5 out of 32 patients (15.6\%) (Fig. 5), and severe stricture in 1 out of 32 patients (3.1\%) (Fig. 6) (Table 3 ).

Our patients were primarily managed through endoscopic or surgical intervention. As 20 patients were managed by endoscopic therapy; 5 patients by balloon

Table 2 Classification of the biliary strictures among our cases according to its site

\begin{tabular}{lll}
\hline Site of stricture & No. & \%/total \\
\hline Anastomotic type & 28 & 87.5 \\
Intrahepatic/non-anastomotic type & 2 & 6.25 \\
Mixed type & 2 & 6.25 \\
Total & 32 & 100.0 \\
\hline
\end{tabular}

dilatation only, 15 patients by balloon dilatation with plastic stent insertion (Fig. 7). Four patients have undergone Roux-en-Y biliary-enteric reconstruction (Fig. 8). Within our cases, MRCP identified the exact locations \& degrees of stenoses in 19 out of 20 recipients managed by endoscopic intervention with only one recipient displaying a false negative result, as MRCP showed moderate anastomotic stricture, while ERCP revealed tight (severe) anastomotic stricture. Consequently, MRCP positive predictive value was $95 \%$, while the negative predictive value was $5 \%$. This means that for every 100 patients who test negative for biliary stricture grading with MRCP, approximately 5 are really negative. So, our study showed good concordance between MRCP and ERCP regarding the diagnosis and grading of post-transplant biliary strictures as 19 out of 20 endoscopically managed recipients diagnosed by MRCP; ERCP revealed the same findings.

Eight patients with a minimal degree of biliary stricture were exposed only for long-term follow-up after their initial MRCP examination (Table 4).

Satisfactory improvement of the biliary stricture was noted in about 16 out of 20 with endoscopic therapy and 2 out of 4 with biliary-enteric reconstruction, as the clinical and laboratory improvement and follow-up MRCP confirmed the diagnosis.

In the present study, on MRCP, nine patients (28.1\%) had a suggestion of biliary leakage presenting with intraabdominal fluid collection traced to the anastomotic site. Comparison of levels of bilirubin from abdominal drainage and serum was used to confirm the diagnosis. All affected patients are completely relieved by intrabdominal pigtail drainage. Two out of these nine patients presented later with postcholangitic hepatic abscesses. During longterm follow-up, we observed intraluminal calcular filling defects in two patients $(6.3 \%)$ about 18 months after transplant, one seen within the native CBD while the other seen within the intrahepatic donor bile duct. 

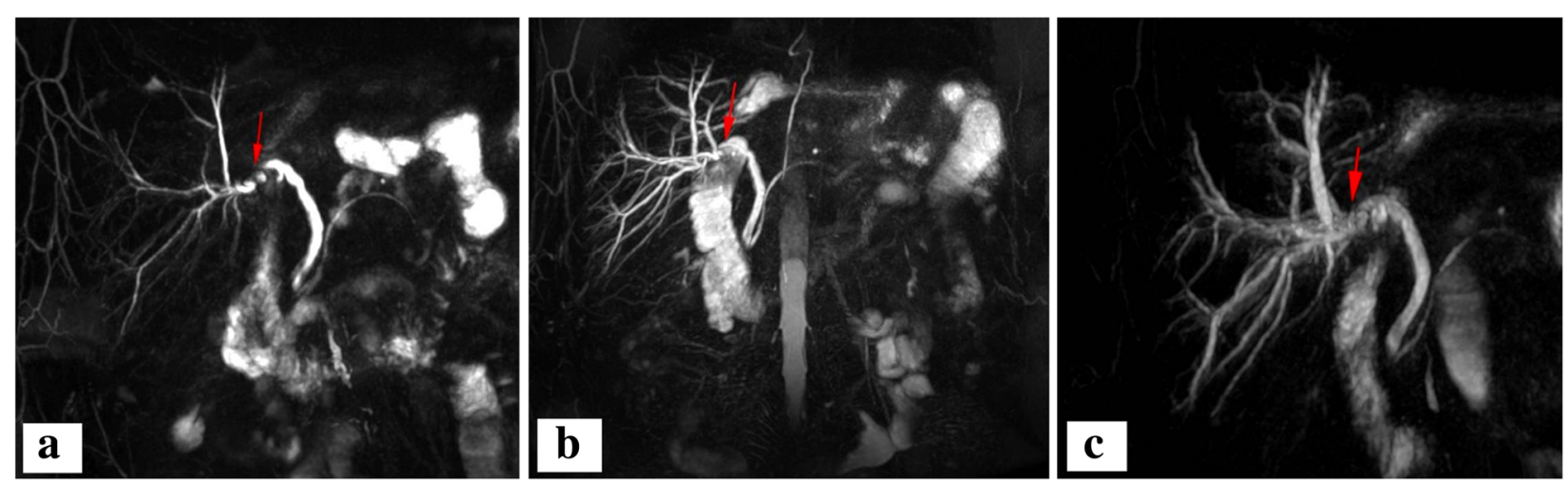

Fig. 5 A 41-year-old male 6 months after liver transplantation with duct-to-duct anastomosis. MRCP 3D MIP reconstructed coronal images showed the presence of anastomotic stricture (red arrow), firstly presented with minimal anastomotic biliary stricture (a) reflecting minimal intrahepatic biliary dilatation followed by two follow-up 3 months interval without management revealed biliary stricture progress to mild degree (b) and moderate degree (c)

\section{Discussion}

A wide spectrum of biliary complications particularly biliary stricture has been seen in post-LDLT recipients. Thus, there is a need for a non-invasive technique that can be used as a screening method in this group. With advances in the imaging technique, MRCP has become a robust, non-invasive potential imaging modality to diagnose biliary strictures [16]. Furthermore, because a contrast medium is not necessary, MRCP presents the biliary tree in its physiologic state. Owing to the inherent contrast provided by the biliary fluid, the bile ducts are visible above and below stenotic site [12].

Superposition of extrahepatic fluid may be a problem during image analysis, especially in patients with frequent bilomas and abdominal fluid collection at the site of the biliary anastomosis. This occurred once in our study; in addition, the main disadvantage of MRCP is its lack of therapeutic ability. So, our study shows that MRCP is an accurate and valuable imaging technique for the assessment of post-liver transplant biliary strictures. In our center, MRCP is performed almost routinely in transplant recipients with abnormal liver enzyme tests or dilated intrahepatic bile ducts (detected by US) to rule out/confirm and to locate biliary strictures.

In the present study, the mean time interval between liver transplantation and presentation with biliary stricture was 7.4 months. This was similar to the mean time interval being 7.1 months in the study performed by Garg et al. [16].

Kyoden et al. [17] reported that the high rate of stricture formation is referred to the surgical techniques which can injure the bile ducts. However, our study showed only one case of intraoperative difficulty of bile duct anastomosis with suture slippage and reanastomosis.

Within our studied cases, from 30 recipients with ductto-duct anastomosis, 28 recipients developed anastomotic biliary strictures, while only two showed non-anastomotic (intrahepatic) strictures, which is in agreement with the study carried out by Shah et al. [18] who reported that
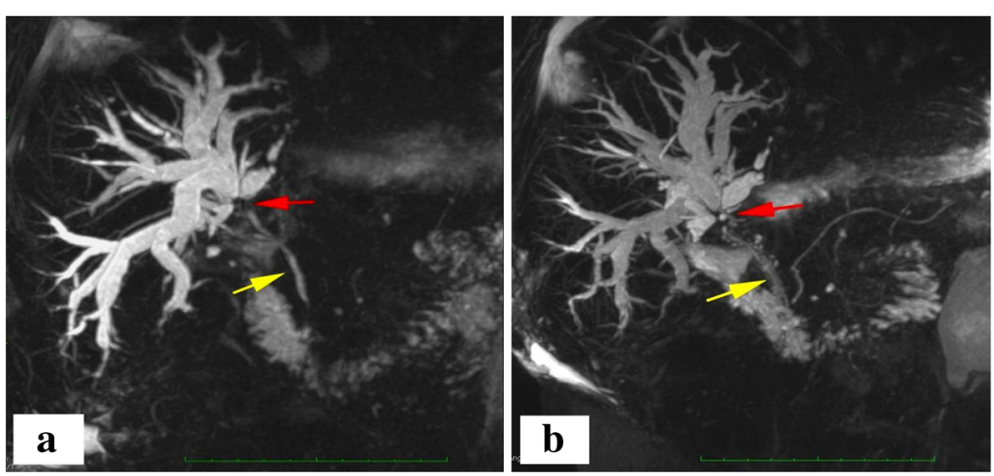

Fig. 6 A 59-year-old male 8 months after liver transplantation with duct-to-duct anastomosis. MRCP 3D MIP reconstructed coronal images showed the presence of severe anastomotic biliary stricture (red arrow) with normal CBD caliber (yellow arrow) (a), managed by CBD stent insertion by ERCP with still persistent stricture with severe intrahepatic biliary dilatation (b) due to slipped stent 
Table 3 MRCP grading of the biliary strictures in our studied cases

\begin{tabular}{lll}
\hline MRCP grading & No. & \%/total \\
\hline Minimal & 7 & 21.9 \\
Mild & 19 & 59.4 \\
Moderate & 5 & 15.6 \\
Severe & 1 & 3.1 \\
Total & 32 & 100.0 \\
\hline
\end{tabular}

duct-to-duct biliary reconstruction has a higher risk of anastomotic stricture formation than biliary-enteric reconstruction.

According to our results, MRCP showed 28 recipients (87.5\%) were diagnosed to have anastomotic biliary strictures, two (6.3\%) had non-anastomotic (Intrahepatic) stricture, and two (6.3\%) had both biliary strictures (mixed). Also, during our study, nine recipients (28.1\%) had a suggestion of biliary leak with two of them presenting later with postcholangitic hepatic abscesses and two $(6.3 \%)$ had filling defects in the biliary tree suggesting the presence of stones. A similar finding was drawn by Garg et al. [16], as in the MRCP study of 34 post-living donor liver transplant recipients reported that 23 recipients $(67.6 \%)$ had anastomotic biliary strictures, nine recipients $(26.5 \%)$ had a suggestion of biliary leak, and four recipients $(11.8 \%)$ had filling defects in the biliary system suggestive of stones or sludge. In three cases, of the total 34 cases, the presence of cholangitis or postcholangitic abscesses was seen in the liver allograft on MRCP.

Our two recipients (6.3\%) with non-anastomotic (intrahepatic) biliary strictures were found to have vascular complications in the form of hepatic artery stenosis and steal phenomenon from the hepatic artery to the gasteroduodenal artery, in addition to one case which was diagnosed before transplantation as primary sclerosing cholangitis, compared with studies carried out by Kochhar et al. [15] and Villa and Harrison [5] who reported that ischemia has been shown to be responsible for the occurrence of non-anastomotic biliary stricture (NAS). Besides ischemia, an immunological cause has also been proposed due to the observation of an increased incidence of NAS in patients with autoimmune hepatitis or primary sclerosing cholangitis.

Our studied recipients with biliary strictures were classified by MRCP into four grades according to the degree of biliary stenosis and subsequent intrahepatic biliary dilatation as the majority of cases presented with a mild degree of biliary stricture (59.4\%), while minimal, moderate, and severe strictures were found to be $21.8 \%$, $15.6 \%, 3.1 \%$ respectively.

Once biliary stricture was diagnosed, the type, number, and degree of strictures were recorded to plan the management. Also, the size discrepancy as well as angulation between donor and recipient bile ducts should be noted [19].

Williams and Draganov [7] showed that the endoscopic therapy is currently the first-line approach to the treatment of post-transplant biliary strictures. ERCP is a widely available and effective procedure and it has relatively limited complications in comparison with surgical intervention which is in agreement with our study as 20 out of 24 managed recipients had undergone endoscopic therapy (by ERCP), compared to only four recipients by surgical intervention (biliary-enteric reconstruction).

In our study, satisfactory improvement of the biliary strictures was noted in about $80 \%$ of managed recipients (16 out of 20 ) by endoscopic therapy, compared with the study carried out by Hsieh et al. [20] who reported that the endoscopic techniques for posttransplant anastomotic biliary strictures attained sustained patency of the biliary anastomoses in about $84.2 \%$ of patients (32 out of 38 ).

Wadhawan and Kumar [19] showed that multiple duct anastomoses and details of each anastomosis should be taken into consideration, as it has implications on the number of stents to be placed. Also, in the case of a single duct anastomosis, the possibility of stricture extending intrahepatically is to be considered as this can convert a single duct anastomosis similar to double duct thus

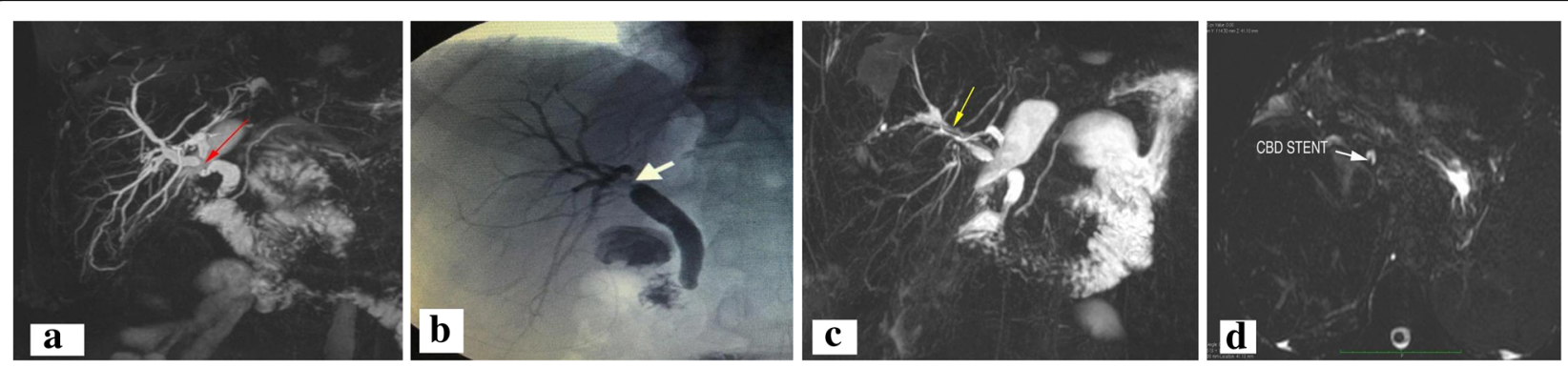

Fig. 7 A 55-year-old male 5 months after liver transplantation with duct-to-duct anastomosis. MRCP 3D MIP reconstructed coronal image showed the presence of moderate anastomotic biliary stricture (red arrow) (a), ERCP was done revealing the same biliary stricture (b), managed by CBD stent insertion (yellow arrow) (c) with MRCP follow-up one month interval showing no intrahepatic biliary dilatation (d) 

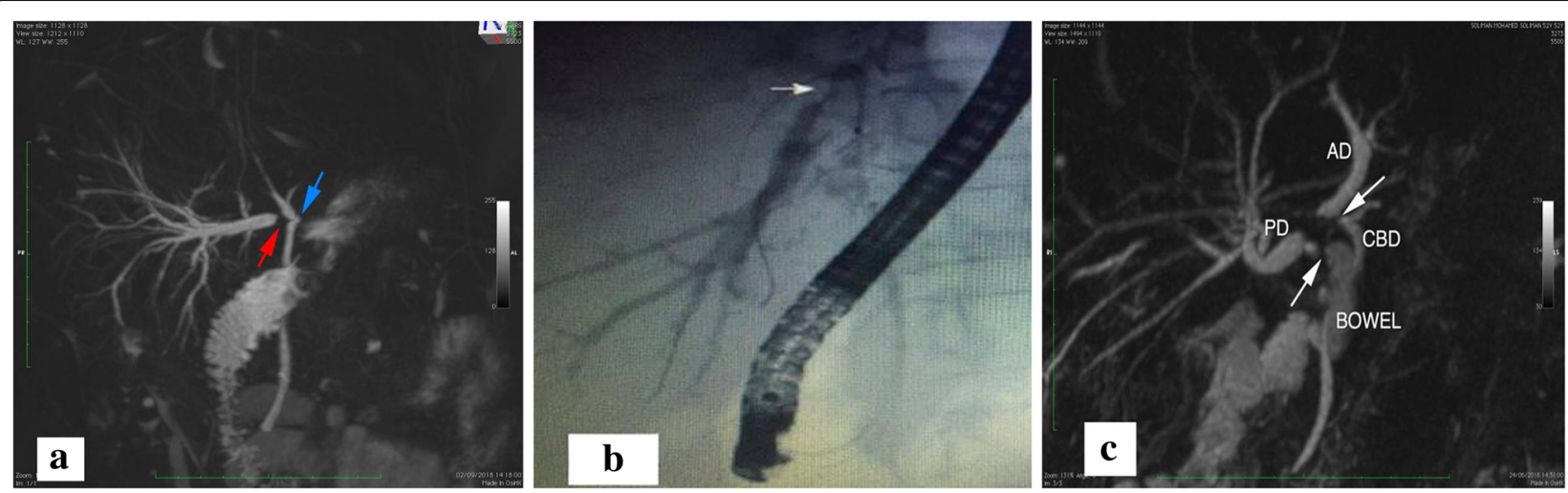

Fig. 8 A 48-year-old female 2 months after liver transplantation with duct-to-duct anastomosis as MRCP 3D MIP reconstructed coronal image showed a kink (blue arrow) at donor anterior bile duct/recipient bile duct anastomosis reflecting minimal anastomotic stricture, associated with moderate intrahepatic biliary stricture at the donor posterior bile duct (red arrow) resulting in post-transplant mixed biliary stricture (a). ERCP revealed a biliary stricture at the posterior sectoral bile duct (white arrow) (b). This patient was managed surgically by biliary-enteric reconstruction after endoscopic therapy failure by stents insertion. Follow up 4 months interval revealed moderate biliary strictures at anastomotic sites with no relief of intrahepatic biliary dilatation (c)

mandating more than one stent. Within our studied cases, two recipients had undergone two CBD stent insertions by ERCP as MRCP diagnosed one of them with complex biliary strictures, anterior bile duct anastomotic kink (minimal degree), and posterior bile duct intrahepatic stricture (moderate degree) with recurrence of stricture about 6 months after stent removal which was defined as biochemical derangement and recurrence of intrahepatic biliary dilatation, shifting management to surgical intervention, which is in agreement with the study carried out by Villa and Harrison [5] who reported that post-transplant cases with narrow or complex anastomotic strictures or stricture extended within the small intrahepatic biliary tree, ERCP guided therapy may fail, and surgical intervention may be required for rescue therapy.

In the present study, two recipients with non-anastomotic (intrahepatic) strictures were managed by endoscopic therapy, one by balloon dilatation only with no improvement of the distal intrahepatic biliary dilatation and the other by balloon dilatation followed by stent insertion with relief of the stricture. Also, within our studied cases, two with mixed (anastomotic and intrahepatic) biliary strictures, as one was managed by ERCP with two stent insertions, its follow-up revealed no improvement in biochemical derangement or

Table 4 Types of management of post-transplant biliary strictures

\begin{tabular}{lllll}
\hline Management & \begin{tabular}{l} 
Endoscopic therapy (ERCP) (20/32) \\
\cline { 2 - 5 }
\end{tabular} & $\begin{array}{l}\text { Biliary- } \\
\text { Balloon } \\
\text { dilatation } \\
\text { only }\end{array}$ & $\begin{array}{l}\text { Balloon dilatation } \\
\text { with stent insertion } \\
\text { anastomosis }\end{array}$ & $\begin{array}{l}\text { Follow- } \\
\text { up }\end{array}$ \\
\hline $\begin{array}{l}\text { No. of } \\
\text { patients }\end{array}$ & 5 & 15 & 4 & 8 \\
\%/total & 15.6 & 46.9 & 12.5 & 25 \\
\hline
\end{tabular}

intrahepatic biliary dilatation, while the other case managed by biliary-enteric reconstruction revealed good improvement with MRCP follow-up.

Our eight cases with MRCP diagnosis of minimal anastomotic biliary stricture $(21.8 \%)$ were exposed only for long-term follow-up without management as only one revealed progress of stricture to mild and then moderate degree with no clinical or biochemical improvement.

The present study and the previous studies show that MRCP is an accurate and useful imaging procedure for the assessment of biliary strictures following living donor liver transplant. The limitation of the present study lies in being a relatively small number of cases examined. In our study, post-LDLT recipients with clinical suspicion of biliary strictures had undergone MRCP to confirm the presence of stricture and record its type and degree, consequently enabling the clinicians with less effort to define the best management plan with better results and limiting the occurrence of post-management complications.

\section{Conclusion}

Our study concludes that MRCP is an effective non-invasive technique to evaluate biliary strictures following LDLT and should be the imaging modality of choice for diagnosis in this setting, in addition to decision-making for the management plan. At present, therapeutic endoscopy plays an important role in the management of anastomotic biliary strictures following liver transplant, while surgical modalities are now reserved for patients in whom endoscopic management failed and for those with multiple inaccessible intrahepatic biliary strictures. So, we hope that MRCP might help in determining the appropriate treatment strategy for recipients with biliary stricture after liver transplantation. 


\section{Abbreviations}

Ass: Anastomotic biliary strictures; DDLT: Deceased donor liver transplantation; ERCP: Endoscopic retrograde cholangiopancreatography; LDLT: Living donor liver transplantation.; LT: Liver transplant; MRCP: Magnetic resonance cholangiopancreatography; NAS: Non-anastomotic biliary strictures; PTC: Percutaneous transhepatic cholangiography

\section{Acknowledgements}

Not applicable.

\section{Authors' contributions}

MSA: put the idea of the research, carried out selection of research cases, prepared the figures for case demonstration, shared in the writing and in the review of the research. RAA: writing the research, sharing in selection of the cases; sharing in figures preparation and follow-up of cases. AES: carried out clinical assessment of the cases and follow-up of cases, supplied the laboratory data, and managed the cases postoperatively. All authors read and approved the final manuscript.

\section{Funding}

No funding obtained for this study from any source.

\section{Availability of data and materials}

The datasets used during the current study are available from the corresponding author on reasonable request.

\section{Ethics approval and consent to participate}

This study is a retrospective study where patient consent was waived by the Research Ethics Board, assuring respect of the confidentiality of the medical record.

\section{Consent for publication}

All patients' consents for publication were obtained by the institutional consent form.

\section{Competing interests}

The authors declare that they have no competing interests.

\section{Author details}

'Radiology Department - National Liver Institute, Menoufia University, Menuofia, Egypt. ${ }^{2}$ Surgery Department - National Liver Institute, Menoufia University, Al Minufya, Egypt.

Received: 4 June 2019 Accepted: 22 July 2019

Published online: 06 September 2019

\section{References}

1. Daniel K, Said A (2017) Early biliary complications after liver transplantation. Clinical liver disease 10(3)

2. Gondolesi G, Varotti G, Florman S et al (2004) Biliary complications in 96 consecutive right lobe living donor transplant recipients. Transplantation. 77(12):1842-1848

3. Macías-Gómez C, Dumonceau J (2015) Endoscopic management of biliary complications after liver transplantation: an evidence-based review. Gastrointest Endosc. 7(6):606-616

4. Londoño M, Balderramo D, Cárdenas A et al (2008) Management of biliary complications after orthotopic liver transplantation: The role of endoscopy. Gastroenterol. 14(4):493-497

5. Villa N, Harrison M (2015) Management of biliary strictures after liver transplantation. Gastroenterology \& Hepatology 11:5

6. Sharma S, Gurakar A, Jabbour N et al (2008) Biliary strictures following liver transplantation: past, present and preventive strategies. Liver Transpl. 14(6): 759-769

7. Williams E, Draganov P (2009) Endoscopic management of biliary strictures after liver transplantation. World J Gastroenterol. 15(30):3725-3733

8. Pasha S, Harrison M, Das A et al (2007) Endoscopic treatment of anastomotic biliary strictures after deceased donor liver transplantation: outcomes after maximal stent therapy. Gastrointest Endosc. 66(1):44-51

9. Verdonk R, Buis C, Van der Jagt E et al (2007) Nonanastomotic biliary strictures after liver transplantation, part 2: management, outcome, and risk factors for disease progression. Liver Transpl. 13(5):725-732
10. Ayoub W, Esquivel C, Martin P et al (2010) Biliary complications following liver transplantation. Dig Dis Sci 55:1540-1546

11. Kohli D, Vachhani R, Shah T, BouHaidar D, Siddiqui M et al (2017) Diagnostic accuracy of laboratory tests and diagnostic imaging in detecting biliary strictures after liver transplantation. Dig Dis Sci. 62:1327-1333

12. Katz $L$, Benjaminovc O, Belinkic A, Gelerd A, Brauna M, Knizhnikc M, Aiznere S, Shaharabanie E, Sulkesf J, Shabtaif E, Pappog O, Atarc E, Tur-Kaspaa R, More E, Ben-Aria Z et al (2010) Magnetic resonance cholangiopancreatography for the accurate diagnosis of biliary complications after liver transplantation: comparison with endoscopic retrograde cholangiography and percutaneous transhepatic cholangiography long-term follow-up. Clin Transplant 24:E163-E169

13. Seo J, Ryu J, Lee $S$ et al (2009) Endoscopic treatment for biliary stricture after adult living donor liver transplantation. Liver Transpl. 15(4):369-380

14. Kitazono M, Qayyum A, Yeh B, Chard P, Ostroff J, Coakley F et al (2007) Magnetic resonance cholangiography of biliary strictures after liver transplantation: a prospective double blind study. J Magn Reson Imaging 25:1168-1173

15. Kochhar G, Parungao J, Hanouneh I, Parsi M et al (2013) Biliary complications following liver transplantation. World J Gastroenterol. 19(19): 2841-2846

16. Garg B, Rastogi R, Gupta S, Rastogi H, Garg H, Chowdhury V et al (2017) Evaluation of biliary complications on magnetic resonance cholangiopancreatography and comparison with direct cholangiography after living-donor liver transplantation. Clinical Radiology https://doi.org/10.1 016/j.crad.2016.12.019

17. Kyoden Y, Tamura S, Sugawara $Y$ et al (2010) Incidence and management of biliary complications after adult-to-adult living donor liver transplantation. Clin Transplant. 24(4):535-542

18. Shah S, Grant D, McGilvray I et al (2007) Biliary strictures in 130 consecutive right lobe living donor liver transplant recipients: results of a Western center. Am J Transplant. 7(1):161-167

19. Wadhawan M, Kumar A (2016) Management issues in post living donor liver transplant biliary strictures. World J Hepatol 8(10):461-470

20. Hsieh T, Mekeel K, Crowell M et al (2013) Endoscopic treatment of anastomotic biliary strictures after living donor liver transplantation: outcomes after maximal stent therapy. Gastrointest Endosc. 77(1):47-54

\section{Publisher's Note}

Springer Nature remains neutral with regard to jurisdictional claims in published maps and institutional affiliations.

\section{Submit your manuscript to a SpringerOpen ${ }^{\circ}$ journal and benefit from:}

- Convenient online submission

- Rigorous peer review

- Open access: articles freely available online

- High visibility within the field

- Retaining the copyright to your article

Submit your next manuscript at $\boldsymbol{\nabla}$ springeropen.com 